\title{
O BALANCED SCORECARD APLICADO À AD- MINISTRAÇÃO PÚBLICA MUNICIPAL: UM ESTUDO NA PREFEITURA DE IBIÚNA, ESTADO DE SÃO PAULO
}

\author{
BALANCED SCORECARD APPLIED TO THE \\ CITY PUBLIC ADMINISTRATION: A STUDY IN \\ THE CITY HALL OF IBIÚNA, SÃO PAULO
}

\section{RESUMO}

O planejamento estratégico produz um resultado articulado, na forma de um sistema integrado de decisões. O Balanced Scorecard permite que haja um alinhamento entre missão, visão e valores, por meio de indicadores que são agrupados em quatro perspectivas que, segundo Niven (2002), na administração pública, são cidadãos, processos internos, aprendizagem e crescimento e orçamento/financeiro. O objetivo deste trabalho foi elaborar o Balanced Scorecard na administração pública no município de Ibiúna, estado de São Paulo, Brasil. A pesquisa tem natureza qualitativa com aplicação do método do estudo de caso, por tratar de situações complexas, contemporâneas e contextualizadas (YIN, 2015). Os resultados indicam que a elaboração do Balanced Scorecard é aplicável ao setor público e permite a consolidação das metas estabelecidas no Plano Diretor, Plano Plurianual, na lei de diretrizes orçamentárias e lei orçamentária anual, aumentando o controle e

Alequexandre Galvez Andrade aleq.galvez@ifsp.edu.br Doutorando em Administração pela Universidad Columbia del Paraguay, UCP, Paraguai. Professor do Instituto Federal de São Paulo. São Roque - SP - BR.

Yohanna Godinho da Silva Moraes yohanna.gsm@gmail.com Bacharel em Administração pelo Instituto Federal de São Paulo. São Roque - SP - BR.

Eduardo Roque Mangini eduardo.mangini@uol.com.br Doutor em Administração. Professor do Instituto Federal de São Paulo. São Roque - SP - BR. a transparência das informações, melhorando o alinhamento com a visão e a missão do município, correspondendo aos preceitos modernos da transparência.

Palavras-chave: Gestão Pública. Planejamento Municipal. Balanced Scorecard.

\begin{abstract}
The strategic planning produces an articulated result, in the form of an integrated system of decisions. The Balanced Scorecard enables an alignment between mission, vision and values by means of indicators that are grouped into four perspectives that, according to Niven (2002), in public administration, the prospects are citizens, internal processes, learning and growth and financial/ budget. The objective of this work was to elaborate the Balanced
\end{abstract}


Scorecard in public administration in the municipality of Ibiúna, state of São Paulo, Brazil. The study has a qualitative nature with the application of the case study method, for dealing with complex, contemporary and contextualized situations (YIN, 2015). The results indicate that the development of the Balanced Scorecard is applicable to the public sector, and allows the consolidation of the goals established in the Master Plan, Multi-year Plan, Law of Budgetary Guidelines and Annual Budgetary Law, increasing the control and transparency of information, improving the alignment with the vision and mission of the municipality, corresponding to the modern precepts of transparency.

Keywords: Public Management. Municipal Planning. Balanced Scorecard.

\section{INTRODUÇÃ̃O}

O Balanced Scorecard (BSC) é uma ferramenta estratégica amplamente usada nas várias organizações privadas (KAPLAN, 2012), cujo foco é a conciliação entre os objetivos aparentemente conflitantes em uma estratégia geral (SMANDEK et al., 2010)in effect, proposing a consistent policy for IP in research and development (R\&D. Além de todo o conjunto de indicadores de desempenho financeiro, o BSC também oferece a mensuração de aspectos intangíveis (RAVAL; KANT; SHANKAR, 2019) bem como retrata a missão e a estratégia em objetivos e medidas, por meio de quatro perspectivas: financeira, do cliente, dos processos internos e do aprendizado e do crescimento (KAPLAN; NORTON, 1992). Como apontado por Agostino e Arnaboldi (2012), a importância do BSC reside em escolher quais as características e como avaliar o desempenho organizacional por meio dos indicadores-chave de desempenho (KPI's). O BSC é uma ferramenta capaz de conectar a estratégia organizacional com tarefas específicas dos colaboradores de uma empresa (ANTONSEN, 2014). Também apresenta, como grande vantagem, a mensuração dos fatores relaciona- dos com a estratégia empresarial (PUNNIYAMOORTHY; MURALI, 2008). Outro fato de destaque é que potencializa a execução do plano estratégico, sejam eles simples ou complexos, de tal forma que são mensurados e recompensados adequadamente (KAPLAN; NORTON, 1996). De fato, o BSC evoluiu de uma simples ferramenta estratégica para um sistema gerencial de desempenho, aumentando, assim, sua importância dentro do contexto organizacional e nos diversos setores da economia (PERKINS; GREY; REMMERS, 2014).

Na gestão pública, essa ferramenta temse mostrado bastante eficiente e eficaz, pois consegue estabelecer elo entre a estratégia estipulada e os indicadores de desempenho da organização (FERNANDES, 2013; NIVEN, 2002). A adaptação do BSC é fundamental, tanto para preservar a identidade do setor público quanto para focar nas necessidades do cidadão (GHELMAN; COSTA, 2006). É indispensável que esse sistema estratégico possa mensurar, de forma adequada, aspectos prioritários em relação aos recursos dos contribuintes e possua orientação para a melhoria da qualidade dos serviços oferecidos e no atendimento ao cidadão (OLIVEIRA; IZELLI, 2018).

Considerando a necessidade premente de melhoria do serviço público, aliado a questões de transparência e eficiência, o uso do planejamento estratégico pode ser considerado como uma possível solução aos gestores municipais, no sentido de melhorar a utilização dos recursos, proporcionar a maior satisfação, e consequentemente, a qualidade na prestação de serviços (COUTO et al., 2016). Diante disso, este trabalho propõe realizar a elaboração do BSC como ferramenta para auxiliar a gestão no setor público municipal com o uso de dados do Plano Diretor, Plano Plurianual (PPA), Lei de Diretrizes Orçamentárias (LDO) e Lei Orçamentária Anual (LOA). Com isso, o problema de pesquisa é: "como elaborar o BSC em organizações públicas municipais?”, e o objetivo principal deste trabalho é elaborar o BSC na administração pública para auxiliar a gestão e o planejamento municipal. A partir do uso do método de 
estudo de caso com observação participante, o estudo teve como objeto a Prefeitura Municipal de Ibiúna com uso de dados secundários que englobam os planos municipais disponíveis no Portal da Transparência em 2018.

O presente artigo é direcionado a pesquisadores e profissionais da administração pública, bem como estudantes e dirigentes do setor público, e está estruturado em cinco partes distintas. A parte seguinte apresenta o referencial teórico, que fornece o embasamento à pesquisa. $\mathrm{Na}$ terceira parte, é descrito o método de pesquisa e os dados da organização pública, seguidos pela quarta parte com a apresentação e a análise dos dados coletados. Por fim, na quinta parte, são apresentadas a conclusão e as perspectivas futuras.

\section{FUNDAMENTAÇÃO TEÓRICA}

A presente seção tem por objetivo examinar a literatura pertinente ao desenvolvimento da pesquisa. Na primeira discussão teórica, abordam-se os aspectos conceituais e as principais características do Balanced Scorecard. Na parte subsequente, discutem-se os conceitos sobre Gestão Pública e os Planos Municipais.

\subsection{BALANCED SCORECARD (BSC)}

O BSC é uma ferramenta estratégica desenvolvida por Kaplan e Norton (1992) que busca alinhar a missão e a visão da empresa com a estratégia implementada, e esse alinhamento consiste em quatro perspectivas: financeira, cliente, processos internos e aprendizagem/ crescimento. O BSC, ao integrar essas quatro perspectivas, auxilia os gestores a entender as relações funcionais cruzadas que podem levar à otimização de soluções e à tomada de decisão mais assertiva (EKMEKÇI, 2014). As quatro perspectivas do BSC, além de permitirem essa otimização da tomada de decisão, promovem a identificação, a racionalização e o alinhamento das iniciativas com a estratégia e a alocação de recursos (GREILING; GREILING, 2010).

Embora o BSC tenha sido desenvolvido como um sistema de medição, essa ferramenta pode ser considerada como um sistema de gerenciamento estratégico (PERKINS; GREY; REMMERS, 2014) e ferramenta de comunicação para as empresas que implementam, integralmente, suas múltiplas capacidades (NIVEN, 2002). Sendo um sistema de controle estratégico que não se restringe, unicamente, aos indicadores financeiros, também aborda as medidas não financeiras para que a organização seja observada em todos os aspectos (INAMDAR; KAPLAN, 2002).

O BSC mantém os indicadores financeiros para a análise do resultado final do desempenho gerencial e corporativo; entretanto, inclui outros indicadores intangíveis que buscam a melhora do clima organizacional (KAPLAN; NORTON, 1992; PUNNIYAMOORTHY; MURALI, 2008). Na perspectiva financeira, são necessárias ações para gerar imagem positiva aos investidores empresariais (CHAVAN, 2009), e são englobados indicadores e métricas relativos ao crescimento da receita, à redução de custos, à melhoria da produtiva e administração e riscos (KAPLAN; NORTON, 1996). Na perspectiva relacionada aos clientes, abrangem questões relativas à participação de mercado, à satisfação dos clientes, bem como indicadores sobre captação e retenção dos clientes (WILSON; HAGARTY; GAUTHIER, 2006). Sobre os processos, o BSC avalia o grau de inovação nos processos de gestão e a qualidade das operações (KAPLAN; NORTON, 1992). Por fim, a perspectiva da Aprendizagem e do Crescimento abrange questões fundamentais para a sobrevivência empresarial, e essa perspectiva ganha importância a partir dos recursos humanos voltados para a geração de novos produtos e vantagem competitiva (PAPENHAUSEN; EINSTEIN, 2006).

Embora o BSC esteja relacionado na mensuração do desempenho financeiro, essa medida não reflete a missão organizacional de entidades públicas e organizações cujo foco principal não é a lucratividade (EKMEKÇI, 2014). Na esfera pública, o BSC deve incluir medidas relacionadas com a excelência na prestação de serviços, 
já que as organizações governamentais devem realizar função social (efetividade) com qualidade na prestação de serviços (eficácia) e com o menor consumo de recursos possível (eficiência) (GHELMAN; COSTA, 2006). Isso é embasado na missão governamental, já que tais organizações são caracterizadas por objetivos políticos e sociais, e, portanto, torna-se necessária a adaptação do BSC para essas instituições (CARMONA; GRÖNLUND; CARMONA, 2003). Esse fato é corroborado por Wilson; Hagarty e Gauthier (2006) que afirma que o BSC é uma prática gerencial e estratégica aceita no setor público, como exemplos a cidade de Charlotte (EUA) implementou o BSC (KAPLAN; NORTON, 2000) e o escritório de auditoria do Texas - EUA (TSAO), que, em 2000, ganhou o prêmio Melhores Práticas (Best Practices) da Associação dos Contadores do Governo - EUA (NIVEN, 2002).

De uma forma geral, o BSC possui, como finalidade, orientar a gestão da organização e quem faz parte dela, utilizando-se de indicadores financeiros e não financeiros para satisfazer os clientes, colaboradores e outros stakeholders da empresa (KAPLAN; NORTON, 1996). Já, na administração pública, o BSC tem como intuito reduzir os custos e melhorar a eficiência operacional dos serviços para a sociedade (FERNANDES, 2013; GHELMAN; COSTA, 2006). Para Niven (2002), o BSC é aplicável ao setor público e dedica um capítulo para explicar os desafios e as adaptações necessárias, sendo a mais significativa a inversão da sequência das perspectivas. Nas organizações públicas, é constituída por cidadãos, processos internos, aprendizagem e crescimento e orçamento, o que determina a importância da execução orçamentária para garantir o cumprimento das metas estabelecidas.

Entretanto, para o BSC formulado obter sucesso - tanto nas organizações privadas, quanto nas públicas e sem fins lucrativos - ele deve ser monitorado por seus criadores, além de atingir todos os níveis organizacionais (WILSON; HAGARTY; GAUTHIER, 2006).

Os mapas estratégicos para administra- ção pública municipal são baseados no Plano Plurianual e Lei Orçamentária Anual. Para isso, são criados indicadores e metas que possam identificar e mensurar os objetivos que pretendem ser atingidos, conforme o planejamento estratégico municipal.

\subsection{PLANEJAMENTO NO SETOR PÚBLICO}

O planejamento estratégico (PE) formulado tanto nas organizações privadas, quanto nas públicas possui, como objetivo, uma melhoria nos resultados, tornando-os mais eficazes, eficientes e efetivos (RIBEIRO et al., 2016). Entre os vários erros e equívocos no âmbito da administração pública, a falta de planejamento formal propicia o aumento da ineficiência, o desperdício de recursos públicos e eleva os custos dos serviços prestados (ERIG; ANTUNES JUNIOR; RAIMUNDINI, 2009).

$\mathrm{Na}$ administração pública, o planejamento estabelecido se dá por meio de documentos formais instituídos pela Constituição Federal: o Plano Diretor, o Plano Plurianual (PPA), a Lei de Diretrizes Orçamentárias (LDO) e a Lei Orçamentária Anual (LOA). A principal dificuldade no setor público é alinhar o processo do planejamento estratégico com o Plano Plurianual e o orçamento. Nessa perspectiva, o BSC ganha destaque, pois pode auxiliar no controle e no acompanhamento das ações desenvolvidas, promovendo a integração e o alinhamento do planejamento municipal e as metas holísticas da cidade como um todo (ERIG; ANTUNES JUNIOR; RAIMUNDINI, 2009). Porém, a mera declaração do uso de ferramentas de gestão, especificamente do BSC, não assegura a eficácia do processo e a garantia de um serviço público de melhor qualidade (SOMERS, 2005).

\subsubsection{Plano Diretor}

Conforme a Constituição Federal de 1988 , em seu artigo $182, \S 1^{\circ}$, o plano diretor, aprovado pela Câmara Municipal, obrigatório para cidades com mais de vinte mil habitantes, 
é o instrumento básico da política de desenvolvimento e de expansão urbana.

O Plano Diretor Municipal tem como finalidade orientar o desenvolvimento e o crescimento do município, sendo revisto a cada dez anos com a participação da população e da câmara de vereadores. A regulamentação do Plano Diretor se deu pela Lei Federal $n^{\circ}$ 10.257/01, que também é denominada de Estatuto das Cidades. De acordo com as diretrizes gerais estabelecidas, propende-se a ater os princípios, os objetivos e os instrumentos da gestão pública municipal.

\subsubsection{Plano Plurianual}

O Plano Plurianual (PPA), previsto no Artigo 165 da Constituição Federal de 1988, corresponde ao instrumento político utilizado para estabelecer metas e objetivos por quatro anos. Sendo realizado por todas as entidades públicas do país, estados e municípios, como obrigação de estabelecer um planejamento estratégico a ser seguido. O PPA aborda as diretrizes, os objetivos e as metas da gestão pública; também apresenta os gastos a serem realizados para esses fins serem atingidos, com o intuito de dar mais transparência sobre a aplicação dos recursos e dos resultados alcançados. O Plano Diretor, em conjunto com o Plano Plurianual, poderá ser utilizado para o alinhamento estratégico da organização pública que pretende implantar o Balanced Scorecard em sua administração.

\subsubsection{Lei de Diretrizes Orçamentárias}

Além de nortear a elaboração da Lei Orçamentária Anual (LOA), baseado no que foi estabelecido no Plano Plurianual (PPA), a Lei de Diretrizes Orçamentárias (LDO) possui, como finalidade, destacar as prioridades da administração pública para o ano seguinte, já que é feita anualmente. Com isso, permite realizar uma junção entre o planejamento de curto prazo (LOA) e o planejamento de médio prazo (PPA).

A LDO também é utilizada como um regulador sobre as metas estabelecidas pelo Pla- no Plurianual, visto que o PPA está mais ligado ao planejamento estratégico, e a LDO à formulação de indicadores e ações pretendidas caso seja elaborado o BSC.

\subsubsection{Lei Orçamentária Anual}

A Lei Orçamentária Anual (LOA) é o planejamento estabelecido em curto prazo que especifica quando e como serão utilizadas as verbas públicas anualmente, com uma estimativa das possíveis receitas e despesas. O Poder Executivo Municipal é o responsável pela elaboração da LOA. Para esse planejamento obter êxito, deve-se apresentar em conformidade com as metas e os objetivos já estabelecidos no PPA, na LDO, e com a Lei de Responsabilidade Fiscal (LRF). A LRF foi criada com o intuito de trazer maior transparência ao planejamento e à gestão pública.

\section{MÉTODO}

O estudo apresenta natureza qualitativa, com a adoção do estudo de caso. A aplicação desse método surge da necessidade de se compreender fenômenos sociais contemporâneos, complexos e contextualizados (YIN, 2015). Essa complexidade do uso do BSC está relacionada à sua própria caraterística de alinhamento entre clientes, fornecedores, parceiros e gestores (KAPLAN; NORTON; RUGELSJOEN, 2010). Outro ponto favorável à escolha do método é por permitir maior profundidade sobre o objeto de estudo (LÖBLER; LEHNHART; AVELINO, 2014; NIVEN, 2002). Ressalta-se que outros estudos que tratam da elaboração e da implementação do BSC também utilizaram o estudo de caso como método (ANTONSEN, 2014; CHAVAN, 2009; PHILBIN, 2011; ROMPHO, 2011)literature focused on the uses and limitations of the Balanced Scorecard in small and medium-sized enterprises (SMEs.

O objeto de estudo foi a Prefeitura de Ibiúna/SP. A cidade de Ibiúna está situada na mesorregião de São Paulo; é considerada um dos maiores municípios em termos de dimen- 
são territorial. Essa delimitação é importante para contextualizar a elaboração do BSC.

A pesquisa foi estruturada em cinco etapas, observando o modelo proposto por (MOTERLE; WERNKE; JUNGES, 2019)'"ISSN":"1678-6483","abstract":"Algumas pesquisas relatam que os gestores das pequenas empresas têm dificuldades para utilizar instrumentos gerenciais da área financeira, o que pode ser influenciado pelo nível de conhecimento dos conceitos relacionados. Nesse sentido, o estudo teve o objetivo de identificar o nível de conhecimento sobre determinados conceitos financeiros por parte dos gestores de micro e pequenas empresas de uma microrregião do Sul de Santa Catarina. A pesquisa se caracteriza como descritiva, com abordagem quantitativa e utilização do procedimento survey para a coleta dos dados de 299 pequenas firmas. Após breve revisão da literatura e evidenciação de estudos anteriores, foram apresentados os dados levantados acerca do nível de conhecimento sobre os conceitos abrangidos. Os resultados destacam que ao menos 55\% dos gestores possuem conhecimento sobre os conceitos pesquisados, mas esse contingente não ultrapassou os $75 \%$ em qualquer dos itens investigados. Dessumiu-se que o menor nível de conhecimento dos gestores se refere ao conceito de liquidez corrente. Por outro lado, os conceitos mais conhecidos pelos gestores estão vinculados à contabilidade financeira (Ativo Circulante, Passivo Circulante e Demonstração do Resultado. Sendo a primeira uma análise documental; nas demais, utilizou-se da observação participante com a participação do secretário de finanças, uma categoria especial de observação, cujo pesquisador não é apenas um observador passivo (YIN, 2015).

$\mathrm{Na}$ primeira etapa, analisou-se o planejamento do município contido no Plano Diretor Municipal, Plano Plurianual (PPA), Lei de Diretrizes Orçamentárias (LDO) e na Lei Orçamentária Anual (LOA). Esse material estava acessível ao público em geral no Portal da Transparência e no site da Câmara Municipal, devido à tempestividade das peças, sendo o pla- no diretor, período de 10 anos, o PPA, 4 anos e a LDB e LOA, anual. Utilizou-se o ano de 2018 para esta pesquisa, por representar o último ciclo orçamentário encerrado.

Na segunda etapa, foram definidos os objetivos estratégicos. Na terceira etapa, os programas de governo foram classificados com os objetivos estratégicos. Na quarta etapa, foi elaborado o Mapa Estratégico. Na quinta parte, foram criados os indicadores de desempenho e, por fim, os resultados foram consolidados e apresentados ao secretário de finanças do município.

\section{RESULTADOS, ANÁLI- SE E DISCUSSÃO}

A partir das análises iniciais e objetivos propostos no PPA, foram obtidas informações que serviram como categorias para a formulação do mapa estratégico e do BSC. O agrupamento das categoriais resultou nas perspectivas. Sendo assim, foi formulado o modelo de BSC proposto por Kaplan e Norton (1992) e adaptado por Niven (2002) para atender à gestão pública, utilizando-se das seguintes perspectivas para a administração pública: cidadãos, processos internos, aprendizagem e crescimento e orçamento/financeiro, conforme a figura 1 . 
Figura 1 - Categorias finais (Perspectivas)

\begin{tabular}{|c|c|}
\hline PPA/LOA/LDB & Objetivo Estratégico \\
\hline \multicolumn{2}{|l|}{ Perspectiva Cidadãos/Sociedade } \\
\hline $\begin{array}{l}\text { 1. Aumentar a oferta de vagas no ensino público na modalidade educação infantil } \\
\text { e fundamental com a construção e reformas de creches, pré-escolas e escolas. }\end{array}$ & \multirow[t]{2}{*}{ Garantir o ensino público com qualidade a todos } \\
\hline 2.Auxilio transporte aos universitários & \\
\hline 3.Manutenção da saúde básica e melhorias ambulatorial e hospitalar & Manter e melhorar a saúde básica \\
\hline $\begin{array}{l}\text { 4.Difusão cultural } \\
\text { 5. Incentivo ao turismo, esporte e lazer }\end{array}$ & Incentivar a difusão cultural, turismo, esporte e lazer \\
\hline $\begin{array}{l}\text { 6. Assistência social: proteção social especial a população carente e } \\
\text { assistência a pessoa com deficiência }\end{array}$ & \multirow{2}{*}{ Garantir à Assistência Social para a População } \\
\hline $\begin{array}{l}\text { 25. Serviços funerários e cemitérios para atender a população carente do } \\
\text { municipio }\end{array}$ & \\
\hline $\begin{array}{l}\text { 7.Infraestrutura urbana } \\
24 . \text { Urbanismo, transporte e projetos } \\
\end{array}$ & \multirow[t]{2}{*}{ Melhorar a Infraestrutura urbana } \\
\hline $\begin{array}{l}\text { Perspectiva Processos Internos } \\
\text { Pernos }\end{array}$ & \\
\hline 8.Desenvolvimento econômico pela promoção da indústria e do comércio & Fomentar o desenvolvimento econômico \\
\hline $\begin{array}{l}\text { 9.Programa de modernização da administração tributária e gestão dos setores } \\
\text { sociais básicos para modernização da gestão pública }\end{array}$ & $\begin{array}{l}\text { Modernizar a administração tributária e a gestão dos setores sociais } \\
\text { básicos da gestão pública }\end{array}$ \\
\hline 12. Manutenção do gabinete do prefeito e dependências & \multirow{8}{*}{$\begin{array}{c}\text { Modernizar as secretarias municipais } \\
\text { Aperfeiçoar o atendimento }\end{array}$} \\
\hline 13. Manutenção da secretaria de governo & \\
\hline 14. Manutenção da secretaria de administração & \\
\hline 15. Manutenção da secretaria de finanças & \\
\hline 17. Manutenção da secretaria de arrecadação & \\
\hline 19. Manutenção da secretaria de agricultura & \\
\hline 21. Administração da secretaria de saúde & \\
\hline \multirow{2}{*}{\multicolumn{2}{|c|}{$\begin{array}{l}\text { 22. Manutenção da secretaria de habitação } \\
\text { Perspectiva Aprendizagem e Crescimento }\end{array}$}} \\
\hline & \\
\hline 16.Pasep (Programa de Formação do Patrimônio do Servidor Público) & Benefícios \\
\hline 12. Manutenção do gabinete do prefeito e dependências & \multirow{8}{*}{$\begin{array}{c}\text { Treinamento } \\
\text { Satisfação Funcional } \\
\text { Incentivar a Qualificação } \\
\text { Implantar à Administração de Desempenho }\end{array}$} \\
\hline 13. Manutenção da secretaria de governo & \\
\hline 14. Manutenção da secretaria de administração & \\
\hline 15. Manutenção da secretaria de finanças & \\
\hline 17. Manutenção da secretaria de arrecadação & \\
\hline 19. Manutenção da secretaria de agricultura & \\
\hline 21. Administração da secretaria de saúde & \\
\hline 22. Manutenção da secretaria de habitação & \\
\hline \multicolumn{2}{|l|}{ Perspectiva Fina } \\
\hline 10. Reserva de contingência & Constituir Reserva de Contingência \\
\hline Lei Orçamentária Anual & $\begin{array}{l}\text { Aumentar os Recursos provenientes do Estado e da União } \\
\text { Aumentar a Base Tributária }\end{array}$ \\
\hline
\end{tabular}

Fonte: elaborado pelos autores.

\subsection{FORMULAÇÃO DO MAPA ES- TRATÉGICO}

A missão e a visão da prefeitura de Ibiúna - SP não estavam definidas, sublinha-se que esse é um dos grandes problemas a ser enfrentado pela administração pública. A dificuldade da identificação da missão no poder público reside no fato de haver forças contraditórias que devem ser enfrentadas para que seja possível desenvolver medidas de desempenho (NIVEN, 2002). O fator moderador para a implantação é o contexto político-ideológico que pode facilitar o equilíbrio no enquadramento de novos papéis para o $\mathrm{BSC}$, aprimorando o alinhamento estratégico (BOBE; MIHRET; OBO, 2017).

Para identificar a missão, procedeu-se à análise documental e foi definida pela frase: "Proporcionar para os cidadãos o desenvolvimento econômico, social e cultural com sustentabilidade, atendendo as necessidades por meio de um trabalho eficiente e eficaz, na busca da qualidade de vida para os munícipes", e a visão: "Trazer ao município uma gestão mais sustentável para aprimorar o atendimento e melhorar a qualidade de vida da população". Ambas as frases foram formadas a partir dos desígnios propostos nos documentos estudados.

Segundo os objetivos destacados no Plano Plurianual do município, o mapa foi dividido conforme as perspectivas propostas para formulação do BSC: cidadãos/sociedade, processos internos, aprendizagem e crescimento e orçamento/financeiro (NIVEN, 2002). A figura 2 demonstra o mapa estratégico. 
Figura 2 - Mapa estratégico Prefeitura Municipal de Ibiúna

\begin{tabular}{|c|c|c|c|c|c|c|}
\hline & Estancia Turistica de & \multicolumn{3}{|c|}{$\begin{array}{c}\text { MAPA ESTRATEGICO } \\
\text { PREFEITURA MUNICIPAL DE IBIÚNA }\end{array}$} & & 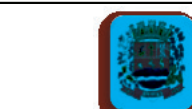 \\
\hline & MISSÃO & \multicolumn{5}{|c|}{$\begin{array}{l}\text { Proporcionar para os cidadãos o desenvolvimento econômico, social e cultural com sustentabilidade, atendendo as necessidades através de } \\
\text { um trabalho eficiente e eficaz, na busca da qualidade de vida para os municipes. }\end{array}$} \\
\hline & VISÃO & \multicolumn{5}{|c|}{ Trazer ao municipio uma melhor gestão sustentável para beneficiar o atendimento e a qualidade de vida da população. } \\
\hline \multirow{7}{*}{ 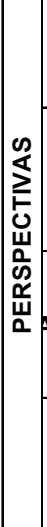 } & Cidadãos/sociedade & $\begin{array}{l}\text { Garantir o ensino público } \\
\text { com qualidade a todos }\end{array}$ & $\begin{array}{l}\text { Manter e melhorar a } \\
\text { saúde básica }\end{array}$ & $\begin{array}{l}\text { Incentivar a difusão cultural, } \\
\text { turismo, esporte e lazer }\end{array}$ & $\begin{array}{c}\text { Garantir à } \\
\text { Assistência Social } \\
\text { para a População }\end{array}$ & $\begin{array}{l}\text { Melhorar a Infraestruture } \\
\text { urbana }\end{array}$ \\
\hline & \multirow[b]{2}{*}{ Processos internos } & & & & & \\
\hline & & $\begin{array}{c}\text { Modernizar as secretarias } \\
\text { municipais }\end{array}$ & \multicolumn{2}{|c|}{$\begin{array}{l}\text { Modernizar a administração tributária e gestão dos } \\
\text { setores sociais básicos da gestão pública }\end{array}$} & $\begin{array}{l}\text { Fomentar o } \\
\text { desenvolvimento } \\
\text { econômico }\end{array}$ & $\begin{array}{l}\text { Aperfeiçoar o } \\
\text { atendimento }\end{array}$ \\
\hline & \multirow[b]{2}{*}{ Aprendizagem e crescimentc } & & & & & \\
\hline & & Treinamentos & Satisfação funcional & Beneficios & $\begin{array}{l}\text { Incentivar a } \\
\text { qualificação }\end{array}$ & $\begin{array}{l}\text { Implantar à } \\
\text { Administração de } \\
\text { desempenho }\end{array}$ \\
\hline & \multirow{2}{*}{ Orçamento/financeiro } & & & & & \\
\hline & & $\begin{array}{r}\text { Aumentar os recursos pro } \\
\text { da Un }\end{array}$ & $\begin{array}{l}\text { enientes do Estado e } \\
\text { ao }\end{array}$ & Aumentar a base tributária & $\begin{array}{l}\text { Reduzir custos e } \\
\text { despesas }\end{array}$ & $\begin{array}{l}\text { Constituir Reserva de } \\
\text { Contingência }\end{array}$ \\
\hline
\end{tabular}

Fonte: elaborado pelos autores.

É possível observar, no mapa estratégico, que há ligação entre cada um dos objetivos propostos nas quatro perspectivas, permitindo explicar a estratégia a ser desenvolvida pela organização e identificando como cada objetivo pode colaborar na execução dos outros objetivos apresentados. No mapa estratégico de 2015 a 2021 do Tribunal de Contas da União, revela-se que esse alinhamento é importante para comunicar todos os níveis da organização pública, permitindo uma compreensão fácil e clara das estratégias, dos indicadores e das metas organizações (BRASIL, 2015).

\subsection{FORMULAÇÃO DO BALANCED SCORECARD}

Definidos os objetivos no mapa estratégico, elaboraram-se, para cada perspectiva, os indicadores medulares para a elaboração do BSC, transformando a estratégia em ação. É desafiador, na administração pública, estabelecer indicadores, devido à longevidade das ações, como as ações na saúde; as sociais e educacionais são de longo alcance. Para vencer esse desafio, recomenda-se estabelecer o objetivo de longo prazo (resultados) e converter em ações de curto e médio prazo (produtos). Dessa forma, medem-se os produtos para atingir os resultados (NIVEN, 2002). No quadro 1, tem-se a perspectiva cidadãos/sociedade e seus indicadores: 
Quadro 1 - Perspectiva cidadãos/sociedade

\begin{tabular}{|c|c|}
\hline \multicolumn{2}{|c|}{ Perspectiva cidadãos/sociedade } \\
\hline Objetivo & Indicadores \\
\hline \multirow{3}{*}{ Garantir o ensino público com qualidade a todos } & $\begin{array}{l}\% \text { de crianças e adolescentes que frequentam as escolas municipais, em } \\
\text { relação ao total de municípes na mesma idade }\end{array}$ \\
\hline & IDEB das escolas municipais \\
\hline & $\%$ de analfabetismo no município \\
\hline \multirow{4}{*}{ Manter e melhorar a saúde básica } & $\%$ de consultas, exames e procedimentos ofertado aos cidadãos \\
\hline & $\begin{array}{l}N^{\circ} \text { de bairros que possuem Unidade Básica de Saúde (UBS), em relação ac } \\
\text { total }\end{array}$ \\
\hline & Tempo para atendimento hospitalar \\
\hline & $\%$ de mortalidade infantil \\
\hline \multirow[b]{2}{*}{ Incentivar a difusão cultural, turismo, esporte e lazer } & Quantidade de eventos culturais e esportivos realizados \\
\hline & $\begin{array}{l}\% \text { de cidadãos atendidos por ações de turismo, esporte e lazer, em relação } \\
\text { ao total }\end{array}$ \\
\hline \multirow[b]{2}{*}{ Garantir à Assistência Social para a População } & $\%$ de famílias carentes no município atendidas, em relação ao total \\
\hline & $\begin{array}{l}\text { \% de pessoas com deficiência que recebem auxílio e tratamento no } \\
\text { município }\end{array}$ \\
\hline \multirow{4}{*}{ Melhorar a Infraestrutura urbana } & $\%$ de estradas rurais e urbanas conservadas, em relação ao total \\
\hline & $\%$ de municípes com acesso a água e esgoto \\
\hline & $\%$ de municípes com acesso a energia elétrica \\
\hline & $\%$ de municípes com acesso a coleta de resíduos \\
\hline
\end{tabular}

Fonte: elaborado pelos autores.

Essa visão baseada no cidadão oportuniza a eficiência de custos, a qualidade do serviço, a coerência e a coordenação de políticas e igualdade de acesso aos serviços (HAMMERSCHMID et al., 2019)we look at the relationship between five key NPM reforms (downsizing, agencification, contracting out, customer orientation and flexible employment practices.

Quadro 2 - Perspectiva processos internos.

\begin{tabular}{|c|c|}
\hline \multicolumn{2}{|c|}{ Perspectiva processos internos } \\
\hline Objetivo & Indicadores \\
\hline \multirow{2}{*}{ Modernizar as secretarias municipais } & $\%$ de processos abertos e deferidos por secretaria \\
\hline & Tempo de resposta aos processos administrativos \\
\hline \multirow{2}{*}{$\begin{array}{l}\text { Modernizar a administração tributária e gestão dos setores } \\
\text { sociais básicos da gestão pública }\end{array}$} & $N^{\circ}$ de projetos elaborados para modernização da gestão pública \\
\hline & \% de realização das ações de modernização \\
\hline \multirow{5}{*}{ Fomentar o desenvolvimento econômico } & $\mathrm{N}^{\circ}$ de empresas que já atuam no município \\
\hline & $\mathrm{N}^{\circ}$ de alvarás concedidos a empresas \\
\hline & PIB per capita \\
\hline & Quantidade de eventos em estímulo ao empreendedorismo \\
\hline & Capacidade da produção agrícola do município \\
\hline \multirow{2}{*}{ Aperfeiçoar o atendimento } & Tempo de atendimento \\
\hline & $\%$ de processos abertos e atendidos \\
\hline
\end{tabular}

Fonte: elaborado pelos autores.

A eficiência operacional deve ser pretendida pelas organizações públicas (FERNANDES, 2013; GHELMAN; COSTA, 2006) e, para a sua garantia, é preciso modernizar. Com o governo aberto, termo criado pela primeira vez na política britânica em 1970, as organizações públicas foram obrigadas a apresentar
Já, na perspectiva de processos internos, contemplou-se, por sua vez, eficiência e eficácia nos objetivos, como se pode observar no quadro 2: 
da vantagem colaborativa, que integra pessoas, empresas e instituições de ensino, amplia as vantagens regionais (KANTER, 1996). No caso de Ibiúna, há uma grande concentração na indústria, por causa do polo industrial instalado na cidade e também no setor agrícola, onde se destaca a

Quadro 3 - Perspectiva aprendizagem e crescimento plantação de hortaliças. Portanto, de um lado, há o aspecto da modernização pública e, do outro, o fortalecimento do arranjo produtivo local. Para suportar esses processos, é imperativo o desenvolvimento e o crescimento das pessoas.

\begin{tabular}{|c|l|}
\hline \multicolumn{2}{|c|}{ Perspectiva aprendizagem e crescimento } \\
\hline Objetivo & \multicolumn{1}{c|}{ Indicadores } \\
\hline Treinamentos & $\mathrm{N}^{\circ}$ de capacitações realizadas \\
\hline Satisfação funcional & $\%$ de satisfação dos servidores \\
\hline Benefícios & $\mathrm{N}^{\circ}$ de benefícios oferecidos \\
\hline \multirow{2}{*}{ Incentivos a qualificação } & $\%$ de servidores com ensino superior \\
\cline { 2 - 2 } & $\begin{array}{l}\mathrm{N}^{\circ} \text { de instituições de ensino superior que possuem parceria com o } \\
\text { órgão público }\end{array}$ \\
\hline \multirow{2}{*}{ Implantar à Administração de desempenho } & $\%$ da meta atingida pelo setor \\
\cline { 2 - 2 } & $\mathrm{N}^{\circ}$ de funcionários que precisam de treinamento \\
\hline
\end{tabular}

Fonte: elaborado pelos autores.

Essa renovação no setor público, estimulada pela característica flexível das mudanças sociais, promove um pensar no aprendizado e no debate (HANSSON; NORN; VAD, 2014) drawing on a case, an evaluation of the Program on Research for Innovation and Renewal in the Public Sector (abbreviated FIFOS. Essa capacidade do ativo intangível em transformar processos, atender às necessidades dos clientes e gerar resultados é decisiva para a transformação das organizações (KAPLAN; NORTON, 2000).

Para a manutenção e a ampliação das ações, é vital garantir os recursos previstos no orçamento público, que é constituído por lei e pode ser reduzido em função do não atendimento das metas fiscais, como o caso da despesa total com pessoal que, no município, não deve exceder a $60 \%$ da receita corrente líquida (BRASIL, 2000). O quadro 4 dispõe da perspectiva orçamento/financeiro e suas medidas:

Quadro 4 - Perspectiva orçamento/financeiro.

\begin{tabular}{|c|l|}
\hline \multicolumn{2}{|c|}{ Perspectiva orçamento/financeiro } \\
\hline \multirow{2}{*}{ Oumentar os recursos provenientes do Estado e da União } & $N^{\circ}$ de projetos e convênios aprovados \\
\hline \multirow{3}{*}{ Aumentar a base tributária } & $\%$ de arrecadação de ISSQN \\
\cline { 2 - 2 } & $\%$ de arrecadação de IPTU \\
\cline { 2 - 2 } & $\%$ de arrecadação total municipal \\
\cline { 2 - 2 } & $\%$ de inadimplência dos contribuintes \\
\hline Reduzir custos e despesas & $\%$ do orçado versus o realizado \\
\hline Constituir Reserva de Contingência & $\%$ destinada do LOA para reserva de contingência \\
\hline
\end{tabular}

Fonte: elaborado pelos autores.

Esse compromisso de acompanhamento é, vigorosamente, alterado pelos meios de comunicação. No caso da cidade de Esslingen - Alemanha, foi criado um fórum on-line para informar e discutir o orçamento, garantindo a participação on-line, o que é fulcral para asseverar a transparência (ROEDER et al., 2005). O acompanhamento desses índices visa a dar uma resposta para a sociedade das medidas que serão ou não implementadas.

\section{CONSIDERAÇÕES FINAIS}

A dificuldade em estabelecer padrões de eficácia e eficiência na administração pública retrata um dos maiores desafios para os gestores, pois nem sempre é possível estipular com facilidade que indicadores conduzem a resultados esperados de longo prazo, por isso é basilar estratificar os resultados de longo prazo em ações de curto prazo. 
Nesta pesquisa, foi elaborado o BSC para a Prefeitura do Município de Ibiúna - SP a partir da definição dos objetivos para cada perspectiva e a elaboração dos mapas estratégicos, indicadores, métricas e objetivos.

Nem sempre a missão e a visão em um órgão público são formais, é preciso aprofundar-se na leitura dos documentos e encadear as ideias com outros pares, para que seja possível extrair a missão e a visão da administração pública. Para acentuar esse problema, há ainda a mudança natural e obrigatória do mandato de prefeitos e o caráter precário de contratação dos agentes públicos comissionados cuja modalidade de contração é baseada na livre nomeação e exoneração.

Estendendo essa evidência da rotatividade no setor público, o próprio BSC pode resultar que gestões futuras tenham acesso ao desempenho da administração, contribuindo para que haja um diagnóstico rápido e confiável dos desafios e das oportunidades para sua gestão.

Essa formalidade dos pilares estratégicos, objetivos, indicadores e metas que compõe o BSC, torna-o uma ferramenta de comunicação que atende aos preceitos modernos de governo aberto, que tem como pilares a transparência, a participação e a colaboração. Além disso, dissemina para todos os interessados as medidas a serem adotadas a fim de atingir as metas estabelecidas nas peças orçamentárias, o que o torna viável, de fácil compreensão e controle.

O presente tema poderá ser explorado em futuras pesquisas a partir dos resultados da implementação do BSC na prefeitura, observando que impactos são revelados com a aplicação da ferramenta, como uma possível base para a formulação do próximo planejamento municipal, e ainda, como essa melhoria, partindo da administração pública municipal, afeta a satisfação dos munícipes.

\section{REFERÊNCIAS}

AGOSTINO, D.; ARNABOLDI, M. Design issues in balanced scorecards : the " what" "and " how" of control. European Management
Journal, v. 30, n. 1, p. 327-339, 2012.

ANTONSEN, Y. The downside of the balanced scorecard: a case study from norway. Scandinavian Journal of Management, v. 30, n. 1, p. 40-50, 2014.

BOBE, B. J.; MIHRET, D. G.; OBO, D. D. Public-sector reforms and balanced scorecard adoption: an Ethiopian case study. Accounting, Auditing and Accountability Journal, 2017.

BRASIL. Senado Federal. Lei Complementar 101, 4 de maio de 2000. Diário Oficial da União, col. 1, p. 1, 5 maio 2000.

BRASIL. Tribunal de Contas da União. Mapa Estratégico TCU (2015-2021). Brasília, 2015.

CARMONA, S.; GRÖNLUND, A.; CARMONA, S. Measures vs actions: the balanced scorecard in Swedish Law Enforcement. Interntional Journal of Operations \& Production Management, v. 23, n. 12, p. 1475-1496, 2003.

CHAVAN, M. The balanced scorecard: a new challenge. Journal of Management Development, v. 28, n. 5, p. 393-406, 2009.

COOPER, D R.; SCHINDLER, P. S. Métodos de Pesquisa em Administração. 12 th ed. Porto Alegre: AMGH Editora Ltda., 2016.

COUTO, C. H. DA M. et al. Planejamento estratégico no setor público: um estudo de caso na prefeitura de Santos Dumont-MG. Revista Brasileira de Gest eo e Engenharia, v. 14, n. 7, p. 128-148, 2016.

EKMEKÇI, Y. A. D. Implementing of Balanced Scorecard: Sample of Turkish Republic Ministry of Youth and Sport. Procedia - Social and Behavioral Sciences, v. 150, p. 754-761, 2014.

ERIG, R.; ANTUNES JUNIOR, J. A. V.; RAI- 
MUNDINI, S. L. Balanced Scorecard na Administração Pública Municipal: um estudo de caso na Prefeitura Municipal de Porto Alegre. Administração Pública e Gestão Social, v. 1, n. 2, p. 151-174, 2009.

FERNANDES, A. G. Balanced Scorecard aplicado à Administração Pública: uma proposta de planejamento Estratégico para o Departamento de Administração e Economia da Universidade Federal de lavras. [S.l.]: Universidade Federal de Lavras, 2013.

GHELMAN, S.; COSTA, S. R. R. Adaptando o BSC para o setor público utilizando os conceitos de efetividade, eficácia e eficiência. In: SIMPÓSIO DE ENGENHARIA DE PRODUÇÃO, 33., 2006, Bauru. Anais [...]. Bauru: UNESP, 2006.

GREILING, D.; GREILING，D. Balanced scorecard implementation in German non-profit organisations. International Journal of Productivity and Performance Management, v. 59, n. 6, p. 534-554, 2010.

HAMMERSCHMID, G. et al. New Public Management reforms in Europe and their effects: findings from a 20-country top executive survey. International Review of Administrative Sciences, v. 85, n. 3, 2019.

HANSSON, F.; NORN, M. T.; VAD, T. B. Modernize the public sector through innovation? A challenge for the role of applied social science and evaluation. Evaluation, v. 20, n. 2, p. 244-260, 2014.

INAMDAR, N.; KAPLAN, R. S. Applying the balanced scorecard in healthcare provider organizations. Journal of Healthcare Management, v. 47, n. 3, p. 179-195, 2002.

KANTER, R. M. Classe Mundial, uma agenda para gerenciar os desafios globais em benefício das empresas e das comunidades. São Paulo: CAMPUS, 1996.
KAPLAN, R., S.; NORTON, D. P. Organização orientada para a estratégia. Rio de Janeiro: [s.n.], 2000.

KAPLAN, R. S. The balanced scorecard: comments on balanced scorecard commentaries. Journal of Accounting \& Organizational Change, v. 8, n. 4, p. 539-545, 2012.

KAPLAN, R. S.; NORTON, D. P. The balanced scorecard: measures that drive performance the balanced scorecard: measures. Harvard Business Review, v. 70, n. 1, p. 71-79, 1992.

KAPLAN, R. S.; NORTON, D. P. Using the balanced scorecard as a strategic management system. Harvard Business Review, v. 74, n. 1, p. 75-85, 1996.

KAPLAN, R. S.; NORTON, D. P.; RUGELSJOEN, B. Managing alliances with the balanced scorecard. Harvard Business Review, 2010

LÖBLER, M. L.; LEHNHART, E. dos R.; AVELINO, A. F. A. Como estão sendo conduzidos os estudos de caso? uma reflexão sobre os trabalhos publicados na área de Administração. In: ENCONTRO DA ANPAD, 38., 2014, Rio de Janeiro. Anais [...]. Rio de Janeiro, 2014.

MOTERLE, S.; WERNKE, R.; JUNGES, I. Conhecimento sobre gestão financeira dos dirigentes de pequenas empresas do Sul de Santa Catarina. RACE - Revista de Administração, Contabilidade e Economia, p. 1-26, 2019.

NIVEN, P. R. Balanced Scorecard Step-byStep: Maximizing Performance and Maintaining Results. New York: John Wiley \& Sons, Inc., 2002.

OLIVEIRA, J. M. de; IZELLI, R. C. Indicadores de Desempenho baseados no Balanced Scorecard: Um modelo adaptado à Administração Pública. Revista Fatec Zona Sul, v. 4, n. 2, p. 37-51, 2018. 
PAPENHAUSEN, C.; EINSTEIN, W. Insights from the Balanced Scorecard Implementing the Balanced Scorecard at a college of business. Measuring Business Excellence, v. 10, n. 3, p. 15-22, 2006.

PERKINS, M.; GREY, A.; REMMERS, H. What do we really mean by "balanced scorecard"? International Journal of Productivity and Performance Management, v. 63, n. 2, p. 148-169, 2014.

PHILBIN, S. P. Design and implementation of the Balanced Scorecard at a university institute. Measuring Business Excellence, 2011.

PUNNIYAMOORTHY, M.; MURALI， R. Balanced score for the balanced scorecard: a benchmarking tool. Benchmarking, v. 15, n. 4, p. 420-443, 2008.

RAMIREZ-ALUJAS, A. V. Gobierno abierto y modernización de la gestión Pública. Tendencias actuales y el (inevitable) camino que viene. Reflexiones seminales. Revista Enfoques, v. 9, n, 15, 2014.

RAVAL, S. J.; KANT, R.; SHANKAR, R. Benchmarking the Lean Six Sigma performance measures: a balanced score card approach. Benchmarking, v. 26, n. 6, p. 19211947, 2019.

RIBEIRO, A. T. V. B. et al. Formalização da estratégia em empresas nascentes de base tecnológica: recursos alternativos na fragilidade do novo. Revista Ibero-Americana de Estratégia, v. 15, n. 4, p. 63-76, 2016.

ROEDER, S. et al. "Public budget dialogue" - An innovative approach to e-participation. In: LECTURE NOTES IN ARTIFICIAL INTELLIGENCE (SUBSERIES OF LECTURE NOTES IN COMPUTER SCIENCE), 2005, Italy. Proceedings [...]. Italy, 2005.

ROMPHO, N. Why the balanced scorecard fails in smes: a case study. International Journal of Business and Management, 2011.

SMANDEK, B. et al. Balanced score card implementation for IP rights management in a public research institution. Measuring Business Excellence, v. 14, n. 4, p. 65-75, 2010.

SOMERS, A. B. Shaping the Balanced Scorecard for use in UK social enterprise. Social Enterprise Journal, v. 1, n. 1, p. 43-56, 2005.

WILSON, C.; HAGARTY, D.; GAUTHIER, J. Results using the balanced scorecard in the public sector Results using the balanced scorecard in the public sector. Journal of Corporate Real Estate, v. 6, n. 1, p. 53-64, 2006.

YIN, R. K. Estudo de Caso: planejamento e métodos. 5. th. ed. Porto Alegre: Bookman, 2015. 